\title{
Editorial
}

\section{Nonlinear Functional Analysis of Boundary Value Problems: Novel Theory, Methods, and Applications}

\author{
Yong Hong Wu, ${ }^{1}$ Lishan Liu, ${ }^{2}$ Benchawan Wiwatanapataphee, ${ }^{3}$ and Shaoyong Lai ${ }^{4}$ \\ ${ }^{1}$ Curtin University of Technology, Perth, WA 6845, Australia \\ ${ }^{2}$ Qufu Normal University, Qufu, Shandong 273165, China \\ ${ }^{3}$ Department of Mathematics, Faculty of Science, Mahidol University, Bangkok 10400, Thailand \\ ${ }^{4}$ Southwestern University of Finance and Economics, Chengdu, Sichuan 610074, China
}

Correspondence should be addressed to Benchawan Wiwatanapataphee; scbww@mahidol.ac.th

Received 24 February 2013; Accepted 24 February 2013

Copyright (C) 2013 Yong Hong Wu et al. This is an open access article distributed under the Creative Commons Attribution License, which permits unrestricted use, distribution, and reproduction in any medium, provided the original work is properly cited.

Nonlinear boundary value problems often arise from scientific research, modelling of nonlinear phenomena, and optimal control of complex systems. In some cases, it is not possible to solve the underlying nonlinear boundary value problems in a closed form, and, for these cases, nonlinear functional analysis can play its role as a tool for obtaining qualitative information about the solution. The primary problems concerned in functional analysis are as follows: does a solution of the boundary value problem exist? If so, is it unique and stable? If the answers to these questions are affirmative, the problem is well posed and the analysis can proceed in a firm base to the next step-construct a method for finding approximate solutions and evaluate the quality of the approximation.

Over the last few decades, intensive research has been carried out worldwide to develop functional analysis theories and methods to tackle complex boundary value problems arising continually from scientific research and modelling of real world phenomena. The papers selected for this special issue represent a typical set of contributions in this field of research. Of course, the selected topics and papers are not an exhaustive representation of the recent development in the field. Nevertheless, they provide novel functional theories and methods as well as rich analysis for many complex boundary value problems with different application backgrounds and we have the pleasure of sharing these research results with the readers. Here, we would like to thank the authors and reviewers of the papers for their excellent contributions. The efforts made by staff of the editorial office of the publishing corporation are also greatly acknowledged.

This special issue contains twenty-nine papers, covering functional analysis of various types of complex boundary value problems for different differential equations and boundary conditions. The particular focus is on fractional order differential equations and partial differential equations, and the boundary conditions include Riemann-Stieltjes integral boundary conditions and nonlocal boundary conditions.

The first set of papers, including nine papers, focus on the study of various complex fractional order boundary value problems.

(i) In the paper titled "Existence of positive solution for semipositone fractional differential equations involving Riemann-Stieltjes integral conditions," based on the fixed-point theorem in a cone, the existence of at least one positive solution is established for a class of semipositone fractional differential equations with Riemann-Stieltjes integral boundary condition.

(ii) In the paper titled "Multiple solutions for a class of fractional boundary value problems," the authors establish conditions for the existence of multiple solutions for a class of fractional bocundary value problems involving left and right Riemann-Liouville fractional integrals of order $0 \leq \beta<1$ by the variational method. 
(iii) In the paper titled "Positive solutions of eigenvalue problems for a class of fractional differential equations with derivatives," the existence of positive solutions for the eigenvalue problem of a class of fractional differential equations is established through establishing a maximal principles and constructing upper and lower solutions.

(iv) In the paper titled "Positive solutions of nonlinear fractional differential equations with integral boundary value conditions," by using the fixed-point theorem in partially ordered set, the authors investigate the existence and uniqueness of the solutions of a boundary value problem involving Caputo fractional derivatives and integral boundary conditions.

(v) In the paper titled "On impulsive boundary value problems of fractional differential equations with irregular boundary conditions," the existence and uniqueness of solutions are established for a class of boundary value problems with irregular boundary conditions and impulsive jumps in the system state and its firstorder derivative.

(vi) In the paper titled "Existence of solutions for nonlinear impulsive fractional differential equations of order $\alpha \in(2,3]$ with nonlocal boundary conditions," the authors established the existence and uniqueness of the solution to a nonlocal boundary value problem for nonlinear impulsive fractional differential equations of order $\alpha \in(2,3]$.

(vii) In the paper titled "Positive solutions of a fractional boundary value problem with changing sign nonlinearity," by investigating the properties of the associated Green function and utilizing the Krasnoselskii fixedpoint theorem in a cone, the authors establish the existence of positive solutions of a boundary value problem for nonlinear fractional differential equations with changing sign nonlinearities.

(viii) In the paper titled "Multiple solutions for a fractional difference boundary value problem via variational approach," the existence of multiple solutions for a fractional difference boundary value problem with a parameter is established within the variational framework.

(ix) In the paper titled "Solutions of sign-changing fractional differential equation with the fractional derivatives," the existence of nontrivial solutions is established for a singular fractional order boundary value problem with a sign changing nonlinear term, through computing the topological degree of a completely continuous field.

The second set of papers, including thirteen papers, focus on the study of various complex partial differential equation boundary value problems.

(i) In the paper titled "On the study of local solutions for a generalized Camassa-Holm equation," the local well-posedness of strong solutions for a nonlinear dispersive model is established in the Sobolev space $H^{s}(R)$ with $s>3 / 2$ by using the pseudoparabolic regularization technique.

(ii) In the paper titled "The well-posedness of solutions for a generalized shallow water wave equation," the authors investigate a generalized shallow water wave equation and discuss the local well-posedness of solutions in the Sobolev space.

(iii) In the paper titled "Blow-up analysis for a quasilinear degenerate parabolic equation with strongly nonlinear source," the authors investigate the properties of the positive solution of the Cauchy problem for a quasilinear degenerate parabolic equation with a strongly nonlinear source and show the existence of a singlepoint blow-up for a large class of radial decreasing solutions.

(iv) In the paper titled "The cauchy problem to a shallow water wave equation with a weakly dissipative term," a shallow water wave equation with a weakly dissipative term is investigated, and the sufficient conditions for the existence of global strong solution are established.

(v) In the paper titled "The local strong and weak solutions for a generalized pseudo-parabolic equation," the wellposedness of local strong solutions for the Cauchy problem of a nonlinear generalized pseudoparabolic equation is established in the Sobolev space.

(vi) In the paper titled "The local strong and weak solutions for a generalized Novikov equation," the Kato theorem for abstract differential equations is applied to establish the local well-posedness of the strong solution for a nonlinear generalized Novikov equation.

(vii) In the paper titled "The asymptotic solution of the initial boundary value problem to a generalized Boussinesq equation," the $L^{2}$ solution of an initial boundary problem for a generalized damped Boussinesq equation is constructed, and the large time asymptotic solution is also obtained.

(viii) In the paper titled "Strong global attractors for 3D wave equations with weakly damping," the authors prove the existence of a global attractor for a 3D weakly damped wave equation in a strong topological space.

(ix) In the paper titled "Regularity and exponential growth of pullback attractors for semilinear parabolic equations involving the Grushin operator," the authors consider a first order nonlinear initial boundary value problem for a semilinear degenerate parabolic equation involving the Grushin operator in a bounded domain and prove the regularity and exponential growth of a pullback attractor in certain function space for the nonautonomous dynamical system associated to the problem.

(x) In the paper titled "A generalization of Mahadevan's version of the Krein-Rutman theorem and applications to $p$-Laplacian boundary value problems," the authors establish a generalization of Mahadevan's version of the Krein-Rutman theorem for a compact, positively 
1-homogeneous operator in a Banach space and then demonstrate its application in establishing the existence of positive solutions for $p$-Laplacian boundary value problems under certain conditions.

(xi) In the paper titled "Existence of solutions for nonhomogeneous A-harmonic equations with variable growth," the authors establish a theorem for the existence of weak solutions for nonhomogeneous A-harmonic equations in subspace and then give three examples to demonstrate its application.

(xii) In the paper titled "Multiple solutions for degenerate elliptic systems near resonance at higher eigenvalues," the authors study the degenerate semilinear elliptic system in an open bounded domain with smooth boundary, and some multiplicity results of solutions are obtained for the system near resonance at certain eigenvalues by the classical saddle point theorem and a local saddle point theorem in critical point theory.

(xiii) In the paper titled "A regularity criterion for the Navier-Stokes equations in the multiplier spaces," the authors establish a regularity criterion in terms of the pressure gradient for weak solutions to the NavierStokes equations in a special class.

The third set of papers, including four papers, deal with several boundary value problems for highly nonlinear ordinary differential equations.

(i) In the paper titled "Positive solutions for second-order singular semipositone differential equations involving Stieltjes integral conditions," the authors investigate the existence of positive solutions for second-order singular differential equations with a negatively perturbed term, by means of the fixed-point theory in cones.

(ii) In the paper titled "Positive solutions for Sturm-Liouville boundary value problems in a Banach Space," the sufficient conditions for the existence of single and multiple positive solutions for a second-order SturmLiouville boundary value problem are established in a Banach space, by using the fixed-point theorem of strict set contraction operators in the frame of the ODE technique.

(iii) In the paper titled "Positive solutions of a nonlinear fourth-order dynamic eigenvalue problem on time scales," the authors study a nonlinear fourth-order dynamic eigenvalue problem on time scales and obtain the existence and nonexistence of positive solutions when $0<\lambda \leq \lambda^{*}$ and $\lambda>\lambda^{*}$, respectively, for some $\lambda^{*}$, by using the Schauder fixed-point theorem and the upper and lower solution method.

(iv) In the paper titled "Bifurcation analysis for a predatorprey model with time delay and delay-dependent parameters," a class of stage-structured predator-prey model with time delay and delay-dependent parameters is considered. By using the normal form theory and center manifold theory, some explicit formulae for determining the stability and the direction of the
Hopf bifurcation periodic solutions bifur-cating from Hopf bifurcations are obtained.

The fourth set of papers focus on finding the approximate and numerical solutions of various complex nonlinear boundary value problems.

(i) In the paper titled "On spectral homotopy analysis method for solving linear Volterra and Fredholm integrodifferential equations," a spectral homotopy analysis method (SHAM) is proposed to solve linear Volterra integrodifferential equations, and some examples are given to test the efficiency and the accuracy of the proposed method.

(ii) In the paper titled "The solution of a class of singularly perturbed two-point boundary value problems by the iterative reproducing kernel method," the authors establish an iterative reproducing kernel method (IRKM) for solving singular perturbation problems with boundary layers and give two numerical examples to demonstrate the effectiveness of the method.

(iii) In the paper titled "A Galerkin solution for Burgers' equation using cubic B-spline finite elements," a Galerkin method using cubic B-splines is set up to find the numerical solutions of Burgers' equation, and the method is shown to be capable of solving Burgers' equation accurately for values of viscosity ranging from very small to very large.

(iv) In the paper titled "Forward-backward splitting methods for accretive operators in Banach spaces," the authors introduce two iterative forward-backward splitting methods with relaxations to find zeros of the sum of two accretive operators in Banach spaces and prove the weak and strong convergence of these methods under mild conditions, and also discuss applications of these methods to variational inequalities, the split feasibility problem, and a constrained convex minimization problem.

Yong Hong Wu Lishan Liu Benchawan Wiwatanapataphee Shaoyong Lai 


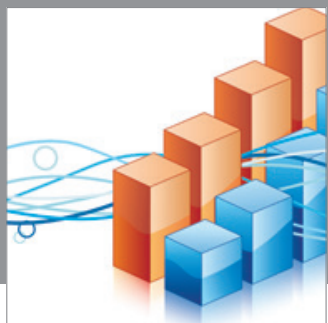

Advances in

Operations Research

mansans

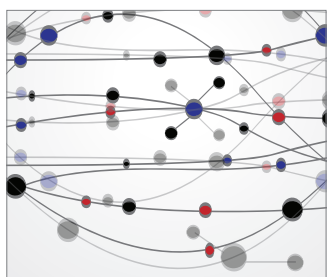

The Scientific World Journal
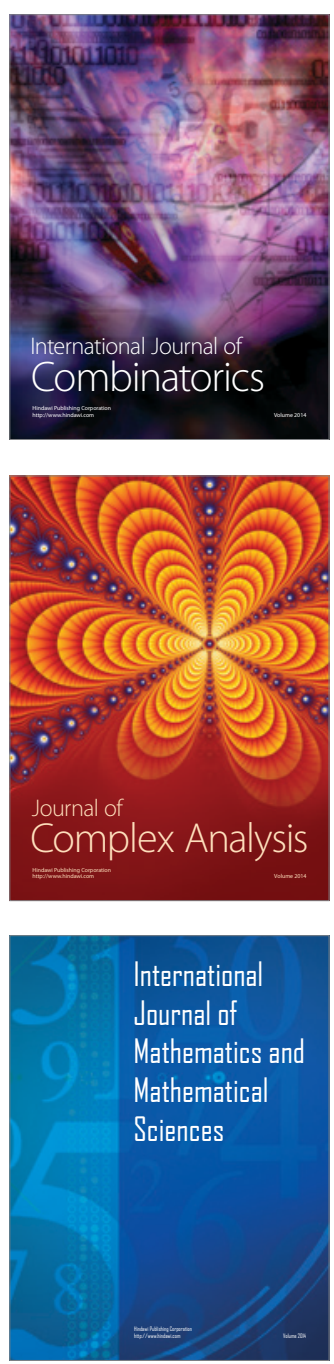
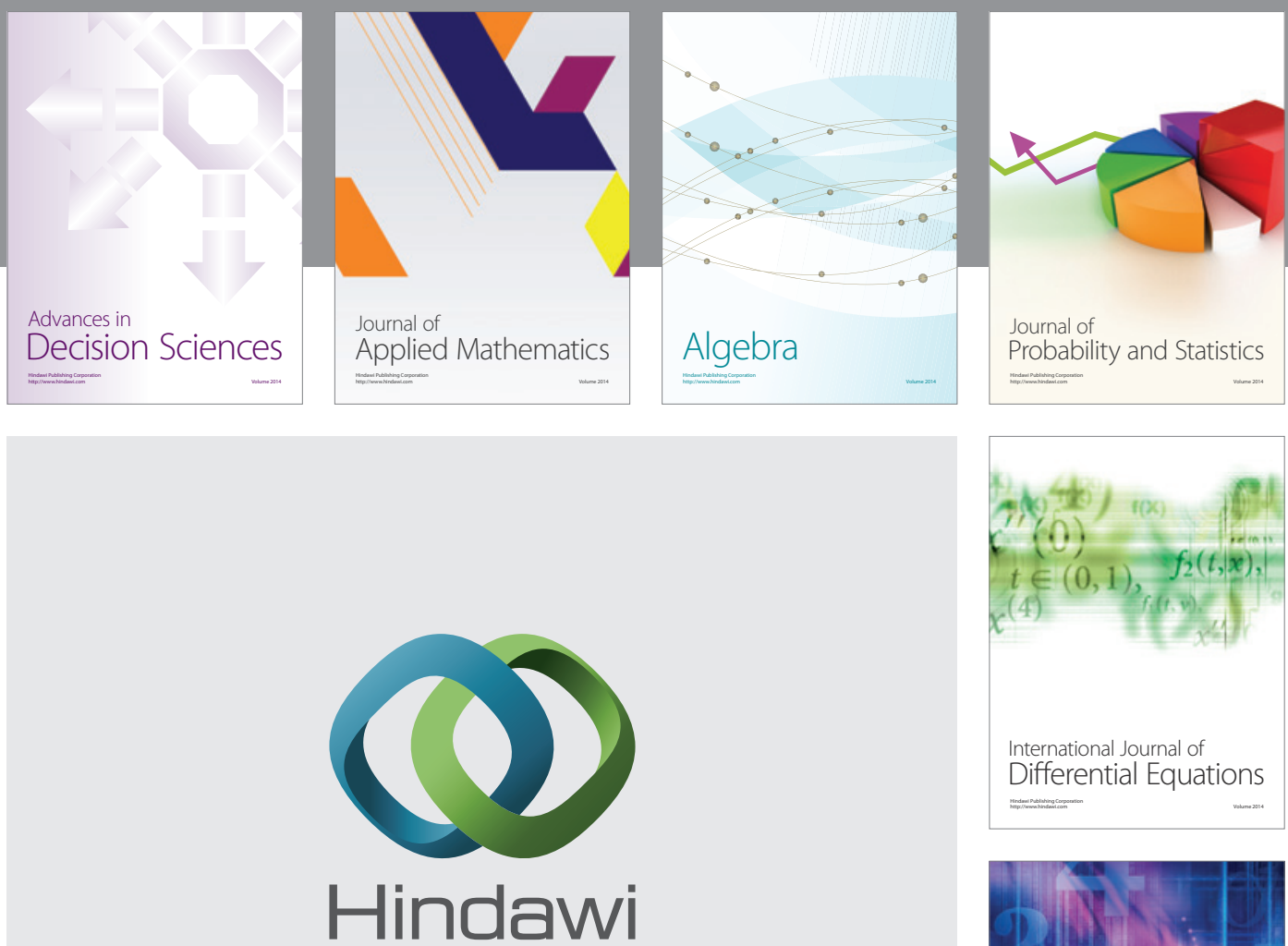

Submit your manuscripts at http://www.hindawi.com
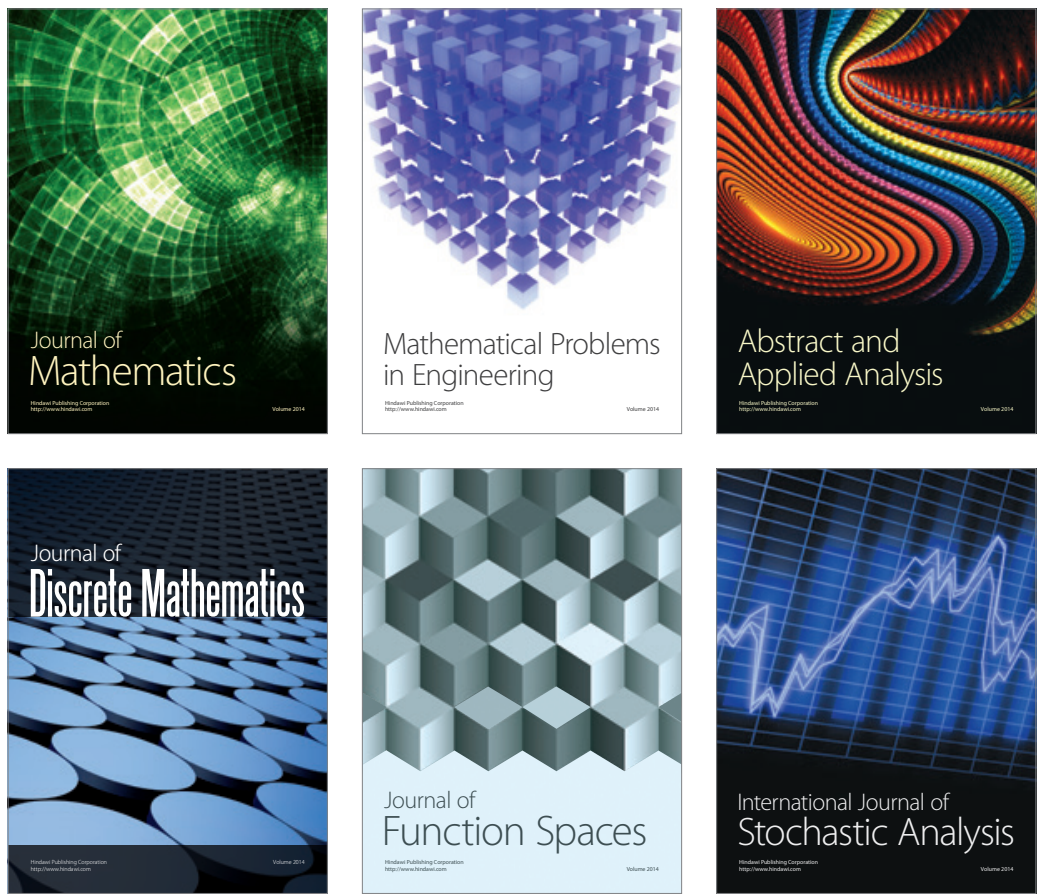

Journal of

Function Spaces

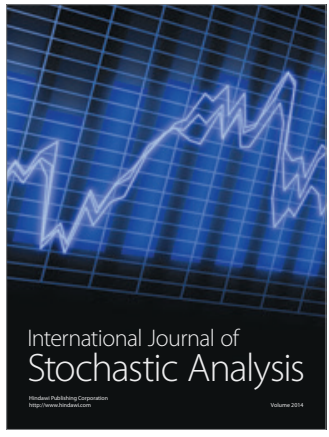

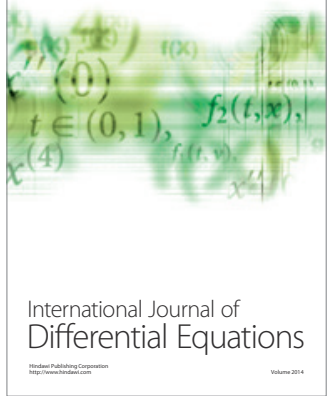
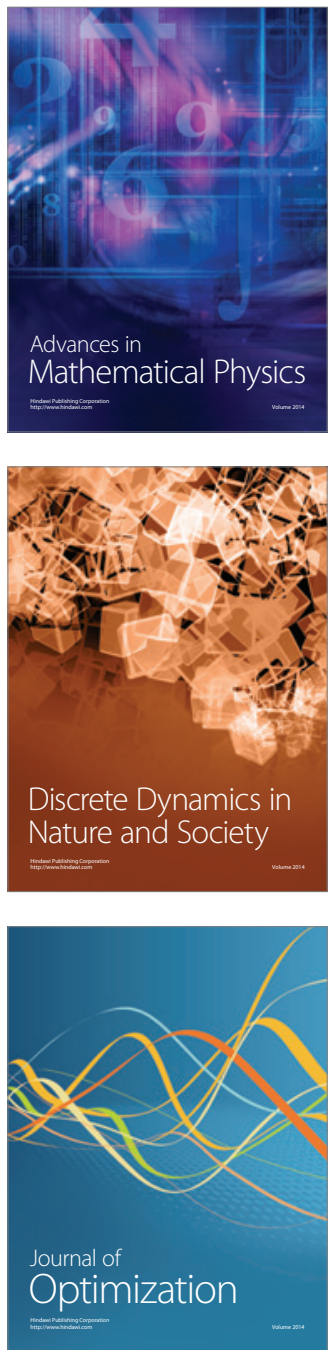